\title{
TWO CASES OF PERICARDITIS ASSOCIATED WITH INFLAMMATORY BOWEL DISEASE
}

\author{
Bilge Tunc ${ }^{1}$, Levent Filik ${ }^{2}$, Aysel Ulker ${ }^{1}$, Erkan Parlak ${ }^{1}$ \\ Turkiye Yuksek Ihtisas Hospital, Ankara, Turkey: Gastroenterology Clinic ${ }^{1}$; Baskent University, Faculty of Medicine, \\ Ankara, Turkey: Department of Gastroenterology ${ }^{2}$
}

\begin{abstract}
Summary: Extraintestinal manifestations are common complications of inflammatory bowel disease (IBD) whereas the association of cardiac disease with IBD is rarely reported. Cardiac manifestations may be diagnosed before, concomitantly or after the diagnosis of the specific type of inflammatory bowel disease. Pericarditis and myocarditis are potentially serious complications. This extraintestinal manifestation developed in one patient concomitantly with onset of intestinal disease. One patient had ulcerative colitis (UC), while other had Crohn's disease (CD). Indomethacin was effective in one and the other patient required prednisone in addition. Chest symptoms in patients with inflammatory bowel disease should be evaluated to exclude myopericardial disease.
\end{abstract}

Key words: Pericarditis; Ulcerative colitis; Crohn's disease

\section{Introduction}

We present here two cases of pericarditis from our group of 600 inflammatory bowel disease (IBD) patients. Cardiac complications of IBD are uncommon; the most frequently reported complication is pericarditis $(70 \%$ of the cases), followed by myocarditis (10\%). Drugs containing 5-aminosalicylic acid have been implicated as the cause of pericarditis in inflammatory bowel disease $(1,2,3,4,6,8,9$, 10).

\section{Case Reports}

Case 1. A 20 year-old-woman with one-year history of non-specific proctitis was admitted to the hospital because of dyspnea, fever, chest pain and chronic diarrhea. She had no remarkable drug history. Investigations revealed that she had pericarditis. The laboratory data with the exception of elevated erythrocyte sedimentation rate (ESR: $62 \mathrm{~mm} / \mathrm{h}$ ), were within the normal ranges. Viral serology showed no evidence of recent viral infection, repeated blood cultures were negative. Results of other immunoserology (latex test, antinuclear antibody, anti-DNA, lupus erythematosus preparation, and complement measurement) were normal. The chest $\mathrm{x}$-ray and electrocardiogram were both negative. Endoscopy, histology demonstrated active Crohn's colitis. She was given mesalamine, prednisolon and her complaints were reduced and losted immediately. The steroid was tapered off. The last echocardiogram con-firmed the dis- appearance of the pericardial effusion. She is currently on a maintenance therapy of mesalamine.

Case 2. A 31 year-old-woman with seven-year history of ulcerative colitis (UC) was admitted to clinic with chest pain, palpitation and bloody diarrhea. Echocardiography confirmed the presence of a moderate pericardial effusion. Collagen-vascular screenings of antinuclear antibody, rheumatoid factor, and complement levels were within normal limits. Viral serology showed no evidence of recent infection. The chest x-ray and electrocardiogram were within normal limits. She was treated with indomethacin and mesalamin and a dramatic improvement was seen in patient's condition.

\section{Discussion}

The causes of cardiac involvement in IBD remain unclear, but the pericarditis must be recognized as a potential extraintestinal manifestation of IBD. That may be diagnosed before, concomitantly or after the diagnosis of the specific type of inflammatory bowel disease. Between $25 \%$ and $36 \%$ of patients with either type of IBD will have at least one extraintestinal manifestation. It is seen more frequently in men and in those with ulcerative colitis. This complication is not related to the activity of the bowel disease $(1,2,3,4,5,6,8,9,11)$. The heart involvement responds well to either nonsteroidal anti-inflammatory drugs or to corticosteroids. Although majority of these cases were interpreted as true extraintestinal manifestations of IBD; a few patients 
were considered to develop cardiac complications as a side effect of treatment (sulfasalazine, mesalamine, and azathioprine). Pericarditis usually starts together with a relapse of the bowel symptoms, or may be the initial manifestation of IBD. Most cases of pericarditis are associated with colonic involvement in both common types of IBD, nevertheless the incidence of pericarditis is slightly higher in UC $(2,3,4,6,8$, $9,11)$. In the specific setting of the two cases reported, pericarditis was considered as an extraintestinal manifestation of IBD. Because both had not used mesalamine, sulfasalasine or other medication soon before diagosis of pericarditis. Accordingly, treatment with 5-aminosalicylic acid (5-ASA) was never stopped and pericarditis has not relapsed during follow-up. Clinical presentations range from asymptomatic pericardial effusions to cardiac tamponade. In the literature, there is a reported case of constrictive pericarditis related to IBD or its treatment (9). Although that patient may have had IBD-associated constrictive pericarditis, her mesalamine use raises the possibility of a druginduced constrictive pericarditis. Additionally, in drug induced pericarditis omission of the 5-ASA therapy was sufficient in a few cases. Pericarditis and myocarditis are rare, but potentially serious complications because there was a few fatal cases (with myocarditis). Both sulfasalazine and the aminosalicylates have been known to cause this complication. Pericarditis as a side effect induced by sulfasalazine or 5-aminosalicylic acid, drugs used in the therapy of these diseases, was first described 15 years ago. Kupferschmidt $\mathrm{H}$ et al. reported that pericarditis was not associated with high activity of bowel disease in all cases (7). In the literature, there is also a reported case of acute pericarditis caused by azathioprine. It is believed that pericarditis is yet another potential manifestation of hypersensitivity to this drug (12). Infliximab, a chimeric antibody targeting tumour necrosis factor is believed to cause cardiac adverse effects but it is still controversial (13). The decision whether pericarditis is a symptom of the underlying disease or a side effect of the drug used for the treatment of the disease is not always easy. Conversely occult inflammatory bowel disease should be excluded when investigating any patient with pericarditis of obscure origin. In conclusion, during the follow-up of patients with IBD, care must be taken concerning extraintestinal cardiac manifestation.

\section{References}

1. Abid MA, Gitlin N. Pericarditis-an extraintestinal complication of inflammatory bowel disease. West J Med 1990;153(3):314-5.

2. Dubowitz M, Gorard DA. Cardiomyopathy and pericardial tamponade in ulcerative colitis. Eur J Gastroenterol Hepatol 2001;13(10):1255-8.

3. Gujral N, Friedenberg F, Friedenberg J, Gabriel G, Kotler M, Levine G. Pleuropericarditis related to the use of mesalamine. Dig Dis Sci 1996;41(3):624-6.

4. Hein L, Petersen CC, Rolighed Larsen JK, Keld DB, Nielsen MB. Pericarditis and inflammatory bowel disease. Ugeskr Laeger 1998;160(32):4651-2.

5. Iaquinto G, Sorrentini I, Petillo FE, Berardesca G. Pleuropericarditis in a patient with ulcerative colitis in longstanding 5-aminosalicylic acid therapy. Ital J Gastroenterol 1994;26(3):145-7.

6. Ishikawa N, Imamura T, Nakajima $\mathrm{K}$ et al. Acute pericarditis associated with 5 aminosalicylic acid (5-ASA) treatment for severe active ulcerative colitis. Intern Med 2001;40(9):901-4.

7. Kupferschmidt H, Langenegger T, Krahenbuhl S. Pericarditis in chronic inflammatory bowel disease: underlying disease or side effects of therapy? Clinical problem solving. Schweiz Med Wochenschr 1996;126(50):2184-90.

8. Molnar T, Hogye M, Nagy F, Lonovics J. Pericarditis associated with inflammatory bowel disease: case report. Am J Gastroenterol 1999;94(4):1099-100.

9. Oxentenko AS, Loftus EV, Oh JK, Danielson GK, Mangan TF. Constrictive pericarditis in chronic ulcerative colitis. J Clin Gastroenterol 2002;34(3):247-51.

10. Patwardhan RV, Heilpern RJ, Brewster AC, Darrah JJ. Pleuropericarditis: an extraintestinal complication of inflammatory bowel disease. Report of three cases and review of literature. Arch Intern Med. 1983;143(1):94-6.

11. Sarrouj BJ, Zampino DJ, Cilursu AM. Pericarditis as the initial manifestation of inflammatory bowel disease. Chest. 1994;106(6):1911-2

12. Simpson CD. Azathioprine-induced pericarditis in a patient with ulcerative colitis. Can J Gastroenterol. 1997;11(3):217-9.

13. Stein RB, Hanauer SB. Comparative tolerability of treatments for inflammatory bowel disease. Drug Saf. 2000;23(5):429-48.

Submitted October 2004. Accepted November 2004.

Dr. Levent Filik, Cemal Gursel Cad. Erk Apt. No: 52/2, Kurtulus, Ankara, 06600, Turkey. e-mail: leventfilik@yahoo.co.uk 